\title{
The Chinese ACL injury population has a higher proportion of small $A C L$ tibial insertion sizes than Western patients
}

\author{
Feilong $\mathrm{Li}^{1,2}$ - Leilei Qin ${ }^{2} \cdot$ Xuan Gong ${ }^{3} \cdot$ Zhenggu Huang $^{1} \cdot$ Ting Wang $^{2} \cdot$ Ziming Liu $^{2} \cdot$ Steve Sandiford ${ }^{4}$. \\ Jianye Yang ${ }^{2}$. Sizheng $\mathrm{Zhu}^{2} \cdot \mathrm{Xi} \mathrm{Liang}^{2} \cdot$ Wei Huang ${ }^{2} \cdot \mathrm{Ning} \mathrm{Hu}^{2}$
}

Received: 16 December 2018 / Accepted: 14 May 2019 / Published online: 20 May 2019

(C) The Author(s) 2019

\begin{abstract}
Purpose The study purpose is to characterize the sizes of the anterior cruciate ligament (ACL) insertion site and intercondylar notch in Chinese patients undergoing ACL surgery. The findings will provide a reference for individualized clinical treatment of ACL rupture.

Methods For this study, 137 patients (102 males, 35 females) with an average age of 30.3 \pm 9.5 years (range 14-52 years) undergoing ACL reconstruction were included. The tibial ACL insertion site length and width and the intercondylar notch width were measured on MRI and arthroscopically using a ruler. Descriptive statistics of the patients, the distribution of the measurements and the differences between males and females were calculated.

Results The ACL tibial insertion size and intercondylar notch width in Chinese patients with ACL injuries, as obtained by MRI and intra-operatively, exhibited significant individual variability. The tibial ACL insertion site had a mean length of $13.5 \pm 2.1 \mathrm{~mm}$ and width of $10.9 \pm 1.5 \mathrm{~mm}$ as measured on MRI and a mean length of $13.3 \pm 2.1 \mathrm{~mm}$ and width of $11.0 \pm 1.6 \mathrm{~mm}$ as measured intra-operatively. The mean intercondylar notch width was $15.2 \pm 2.4 \mathrm{~mm}$ on MRI and the mean length was $15.0 \pm 2.5 \mathrm{~mm}$ intra-operatively. The inter-rater reliability between MRI and intra-operative measurements confirmed that the two methods were consistent. In $65.7 \%$ of individuals, the ACL tibial insertion length was $<14 \mathrm{~mm}$.

Conclusion The distribution of tibial footprint size in Chinese patients is different from that in Western populations. There is a higher proportion of subjects with a tibial footprint size $<14 \mathrm{~mm}$ among Chinese patients with ACL injury. Therefore, great care should be taken when treating this population with the double-bundle technique or larger graft options.
\end{abstract}

Level of evidence IV.

Keywords Anterior cruciate ligament (ACL) $\cdot$ Intercondylar notch width $\cdot$ Insertion size

\section{Introduction}

Due to its unique anatomical structure and effect, rupture of the anterior cruciate ligament (ACL) is relatively common, with the mainstay of treatment being ACL reconstruction

$\mathrm{Ning} \mathrm{Hu}$

huncqjoint@yeah.net

1 Department of Orthopedics, The Dazu District People'S Hospital, Chongqing 402360, China

2 Department of Orthopedics, The First Affiliated Hospital of Chongqing Medical University, Chongqing 400016, China

3 Department of Outpatient, Chongqing General Hospital, Chongqing 400013, China

4 Department of Orthopedics, Queen Elizabeth Hospital, Bridgetown, Barbados
(ACL-R). In recent years, anatomical and biomechanical considerations have resulted in the preference of surgery to restore the anatomy of the ACL $[7,41]$. Many anatomical and biomechanical studies have defined the characteristics of the native state of the ACL, which is housed in the femoral intercondylar notch [48]. Functionally, it has two distinct bundles [49], the anteromedial (AM) bundle and the posterolateral (PL) bundle. However, some instances showed that the ACL insertion site or intercondylar notch size may be too small to accommodate both grafts in a double-bundle (DB) reconstruction [31, 36, 41]. These factors have influenced the selection of grafts and techniques, resulting in a more individualized approach to ACL-R [22, 43].

Specifically, some researchers have suggested that in patients with a tibial insertion site $<14 \mathrm{~mm}$ in length and a notch $<12 \mathrm{~mm}$ in width, the native anatomy may be better 
reproduced with single-bundle (SB) ACL-R [15, 28-30, 44]. The proportion of individuals with an ACL tibial footprint size $<14 \mathrm{~mm}$ in length was reportedly low, varying between $3.6 \%$ and $10.6 \%$ in Western patient populations [22, 39, 47]. However, recent data revealed that the distribution of the size of the ACL tibial footprint widely varied among different ethnic groups; for example, there is a high proportion $(53.5 \%)$ of South Korean females with a tibial footprint length $<14 \mathrm{~mm}$ [27]. We assert that ACL anatomical differences may also occur in Chinese patients, as the ACL tibial footprint in the Chinese population has yet to be thoroughly investigated [27, 34].

Some attempts have been made to address those considerations in similar populations, while there are still limitations, such as relatively small sample sizes, cadaveric specimens of elderly persons, and osteoarthritic patients undergoing total knee arthroplasty [17, 23, 26, 27]. Moreover, considering the previous study showing that patients who have suffered a non-contact ACL injury had a significantly smaller ACL volume in their contralateral knee than the volume of gender-, height-, age-, and weight-matched normal controls [6], and it is possible that a link exists between ACL size and injury risk [45]. Anatomical ACL data may need to be re-evaluated in the Chinese population of patients with ACL injury. Therefore, the following hypotheses are proposed:

1. The ACL insertion site and intercondylar notch size have significant size differences compared to those in Western populations.

2. A higher proportion of ACL insertions is $<14 \mathrm{~mm}$ in Chinese patients with ACL rupture than in Western patients.

The purpose of this study was to identify the ACL insertion site and intercondylar notch size in Chinese patients undergoing ACL surgery. The data will provide a reference for individualized ACL graft production, technical modifications for treatment of Chinese patients with ACL rupture and a clear determination for choosing between SB ACL-R or DB ACL-R in the Chinese population of patients with ACL injuries.

\section{Materials and methods}

The study was carried out from November 2017 to July 2018. All methods were performed in accordance with the ethical guidelines. Informed consent was obtained from all participants.

The main inclusion criteria were recent patients with ACL rupture and reconstruction within 3 months after the injury. The exclusion criteria were as follows: patients with high-energy injuries, such as car accidents and falls, patients with multiple ligamental injuries, patients with severe osteoarthritis (grade III or higher), patients with tuberculosis of the knee, suppurative arthritis, rheumatoid arthritis, or with tumour, or those who underwent previous notchplasty [17, $26,47,48]$. Patients with concomitant injuries such as a meniscus tear or an MCL rupture were not excluded because the notch width and insertion site size of the ACL were not influenced.

One hundred and thirty-seven patients (102 males, 35 females) with an average age of $30.3 \pm 9.5$ years (range $14-52$ years) were included in this study. All patients underwent preoperative MRI and ACL-R at the First Affiliated Hospital of Chongqing Medical University.

\section{Preoperative MRI measurement}

Patients were scanned using a 1.5-T magnet (Signa HDxt, GE Medical System, USA). Images were acquired in 4-mm slices using a flexible phased-array coil and clinically established MRI sequences at the Radiology Department. These views were obtained with the patient in the supine position and the knee in extension. T1-weighted fast spin-echo (FSE) images were obtained in all patients (TE, min full; TR, $760 \mathrm{~ms}$; matrix size, 512*512; bandwidth, $31.25 \mathrm{kHz}$; field of view, $480 \mathrm{~mm}$; slice thickness, $4 \mathrm{~mm}$; spacing, $0.5 \mathrm{~mm}$ ). T2-weighted fast recovery with fast spin-echo (FRFSE) sequence images were also obtained in each patient (auto TR, $2820 \mathrm{~ms}$; echo train length $(18 \mathrm{~mm})$; matrix size, $512 * 512$; bandwidth, $31.25 \mathrm{kHz}$; field of view, $480 \mathrm{~mm}$; frequency, $228 \mathrm{kHz}$; slice thickness, $0.5 \mathrm{~mm}$; spacing, $1 \mathrm{~mm}$ ). In the coronal and sagittal planes, the images were oriented with the same anatomical alignment as the ACL and the femoral notch were, which allowed for a clear and predictable recognition of partial ACL tears and femoral notch shapes.

Currently, MRI is used not only for diagnosis but also for the measurement of the size and inclination angle of the ACL, as well as intercondylar notch size $[1,26,46]$. These data were compared with the intra-operative measurements performed by the surgeon using a specialized ruler and demonstrated high intra-rater reliability [46]. Single coronal and single sagittal proton density images were selected. The image with the most suitable sequence for ACL insertion size measurement was determined as the image that illustrated the ruptured ACL fibre attachment to the tibia [1]. The distance between the most anterior and most posterior fibres of the ACL attachment was determined to measure the exact length of the tibial ACL insertion site (Fig. 1a). The distance between the most medial and the most lateral portion of the ACL attachment was measured as the exact width of the tibial ACL insertion site (Fig. 1b). The coronal image of the most suitable sequence for intercondylar notch width measurement was the initial cut, which showed a clear 


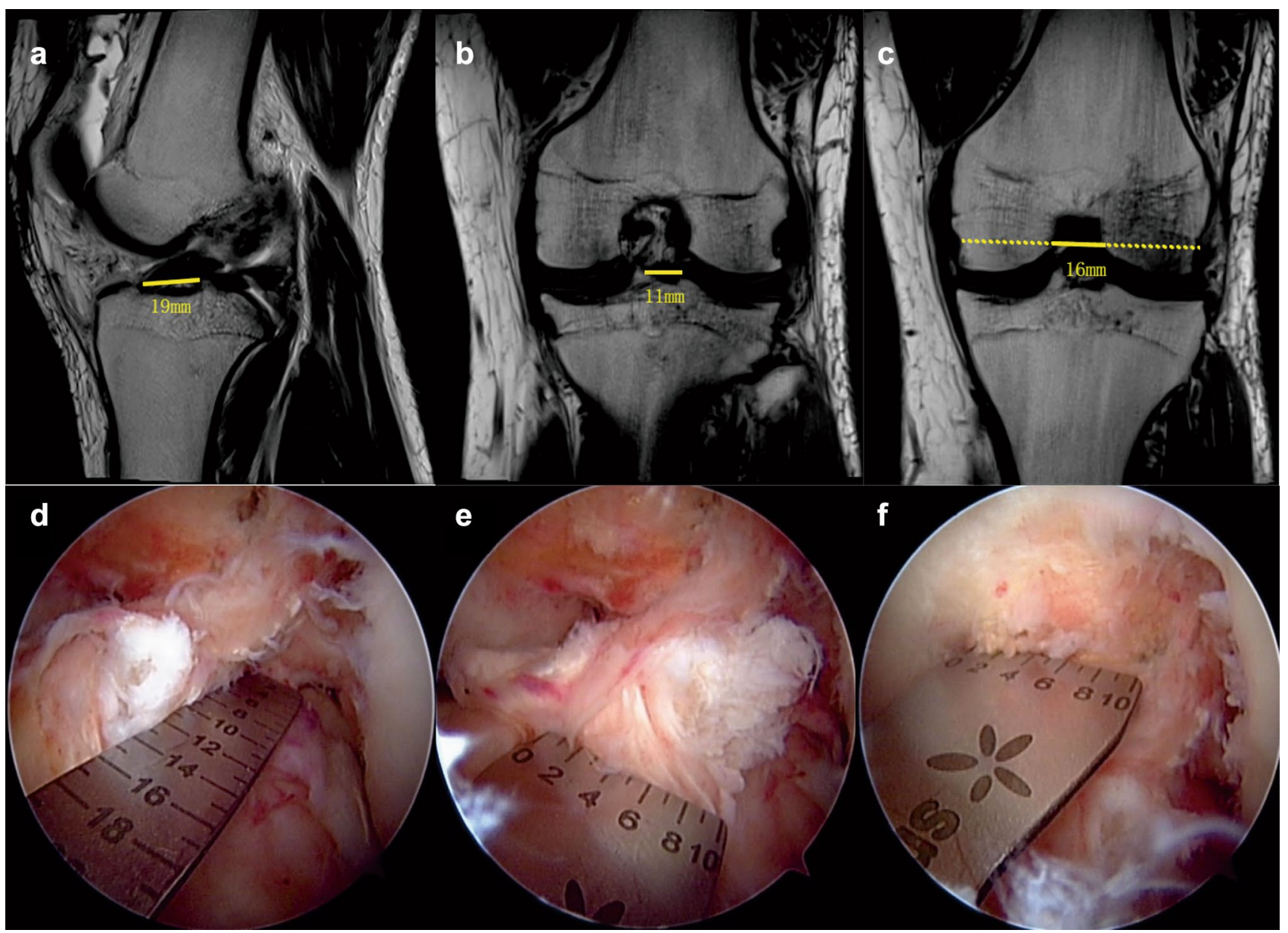

Fig. 1 The nether pictures are arthroscopic views of a knee in flexion. The length (d) and width (e) of the notch (f) are measured using an arthroscopic ruler. A T2 proton density-weighted sequence of knee MRI showing a rupture of the ACL and the knee intercondylar notch.

and continuous image of the medial and lateral condyles (Fig. 1c) [26]. The initial image had a clear notch shape and a clear popliteus tendon sulcus of the lateral condyle. A line on the popliteus tendon sulcus was drawn. This line paralleled the lowest points of the cartilage surface of the medial and lateral condyles. The distance between the line and the crossing point of the medial and lateral condyles was measured as the intercondylar notch width [26]. A blinded observer in the Department of Orthopaedic Surgery (L.F.L.) performed the radiographic evaluations. The measurement was performed using a straight line and was rounded to the nearest $1 \mathrm{~mm}$ (Fig. 10). These results were measured three times, and the average value was used and also rounded to the nearest $1 \mathrm{~mm}$.

\section{Intra-operative measurement}

The intra-operative measurements were made by the senior surgeon. The three accessory medial, anterolateral, a The yellow line signifies the tibial ACL insertion site length. b The yellow line signifies the tibial ACL insertion site width. c The full yellow line signifies the knee intercondylar notch width

and anteromedial arthroscopic portals were established, which allowed nearly parallel-course positioning of the arthroscopic ruler with the tibial ACL remnant fibres and notch. A previous study demonstrated that the three-portal approach could achieve optimal arthroscopic visualization of the ACL insertion sites and intercondylar notch and had excellent accuracy for measuring the sizes of the ACL insertion sites and the intercondylar notch [15, 28, 46, 48]. The surgeon started with routine diagnostic arthroscopy of the knee and initial resection of the disruptive fat pad. The notch and the ACL stumps were clearly visualized [2]. To measure the ACL tibial insertion site, meticulous dissection of the distal remnant tibial ACL fibres was performed with care to preserve the borders of the insertion sites dissected to obtain the best view. Measurements were obtained of the native ACL tibial insertion site length, mid-width with a flexible, reusable, arthroscopic ruler (Smith and Nephew Endoscopy, Andover, Massachusetts), which was graded in millimetres (Fig. 1d, e). 
Measurements of the intercondylar notch, specifically the width at the middle of the notch, were then obtained using a ruler [29] (Fig. 1f). The senior surgeon (N.H.) performed all the operations and finished intra-operative measurements together with the other surgeons.

This clinical trial was approved by the Institutional Review Board and Hospital Ethics Committee of the First Affiliated Hospital of Chongqing Medical University (2017-191).

\section{Statistical analysis}

An a priori sample size calculation was performed for the primary aim. The variables used for the calculation were based on findings of previously reported studies on notch and insertion site sizes (intercondylar notch width: $15.7 \pm 2.6 \mathrm{~mm}$; length of the tibial ACL insertion site: $15.36 \pm 2.33 \mathrm{~mm}$; width of the tibial ACL insertion site: $11.03 \pm 1.77$; significance: 0.05 ; power: 0.9$)$ [23, 26]. This analysis showed that a minimum of 70 subjects should be included in the study. Descriptive statistics were calculated for the ACL tibial insertion site length and width, the notch width at the middle, and the patient age, height, and weight. The mean values, standard deviations, and ranges of measurements were evaluated. The frequency distributions, especially for the ACL tibial insertion size and notch width, were calculated. Differences in males and females were determined through an unpaired $t$ test. Additional Bland-Altman plots were utilized to detect the agreement between the MRI and intra-operative measurements. All statistical analyses were performed with IBM SPSS version 23.0 software (SPSS Inc., an IBM Company, Chicago, Illinois, USA). The level of significance was determined at $P<0.05$.

Table 1 Demographic data

\begin{tabular}{lrrll}
\hline & Mean & \multicolumn{1}{c}{ SD } & Minimum & Maximum \\
\hline Age (years) & 30.3 & 9.5 & 14 & 52 \\
Height $(\mathrm{cm})$ & 170.1 & 8.3 & 138 & 187 \\
Weight $(\mathrm{kg})$ & 71.3 & 13.8 & 40 & 110 \\
BMI $\left(\mathrm{kg} / \mathrm{m}^{2}\right)$ & 24.6 & 4.1 & 16.2 & 45.4 \\
Males $(\%)$ & 74.5 & & & \\
\hline
\end{tabular}

\section{Results}

Age and anthropometric data, including height and weight of the included study subjects $(n=137)$, are given in Table 1. The mean age of the 137 patients evaluated was $30.3 \pm 9.5$ years (14-52 years), with a mean height and mean weight of $170.1 \pm 8.3 \mathrm{~cm}(138-187 \mathrm{~cm})$ and $71.3 \pm 13.8 \mathrm{~kg}(40-110 \mathrm{~kg})$, respectively. A total of 102 patients were men, and 35 were women.

MRI measurements of ACL length and width at the tibial attachment and of the intercondylar notch width, as well as the intra-operative measurements using an arthroscopic ruler are given in Table 2. The average ACL length and width at the tibial attachment on MRI were $13.5 \pm 2.1 \mathrm{~mm}$ and $10.9 \pm 1.5 \mathrm{~mm}$, respectively. The average intercondylar notch width was $15.2 \pm 2.4 \mathrm{~mm}$. The anthropometric measurements are also shown in Table 2.

As expected, the Bland-Altman plot between the MRI and intra-operative measurements also showed excellent agreement (Fig. 2).

Table 3 shows the comparison of the gender subgroups regarding the notch width and ACL insertion site width and length. In terms of gender-related differences, the average male intercondylar notch width was significantly larger $(15.5 \pm 2.4 \mathrm{~mm})$ than that of the female knees $(14.5 \pm 2.2 \mathrm{~mm})(P<0.05)$, and there were no significant gender differences in ACL insertion size.

The tibial insertion site length was variable. The distributions of the recorded lengths and widths are shown in Fig. 3a, b. On the tibial side, more than half $(65.7 \%$ of the total sample) had an insertion site length that was smaller than $14 \mathrm{~mm}$. The proportion of insertion sites between 14 and $16 \mathrm{~mm}$ was $21.9 \%$. The proportion of insertion sites greater than $16 \mathrm{~mm}$ was $12.4 \%$. The distributions of the width of the intercondylar notch are shown in Fig. 3c. The proportion of individuals with an intercondylar notch width $<14 \mathrm{~mm}$ was $32.1 \%$. A total of $22.6 \%$ had an insertion site between 16 and $18 \mathrm{~mm}$. A total of $16.1 \%$ had an insertion site greater than $18 \mathrm{~mm}$.
Table 2 Descriptive statistics $(n=137)$

\begin{tabular}{|c|c|c|c|c|c|c|c|c|}
\hline & \multicolumn{4}{|l|}{ MRI } & \multicolumn{4}{|c|}{ Arthroscopic } \\
\hline & Minimum & Maximum & Mean & SD & Minimum & Maximum & Mean & SD \\
\hline Tibial insertion length & 9 & 20 & 13.5 & 2.1 & 9 & 19 & 13.3 & 2.1 \\
\hline Tibial insertion width & 8 & 15 & 10.9 & 1.5 & 8 & 15 & 11.0 & 1.6 \\
\hline Notch width & 9 & 21 & 15.2 & 2.4 & 8 & 20 & 15.0 & 2.5 \\
\hline
\end{tabular}



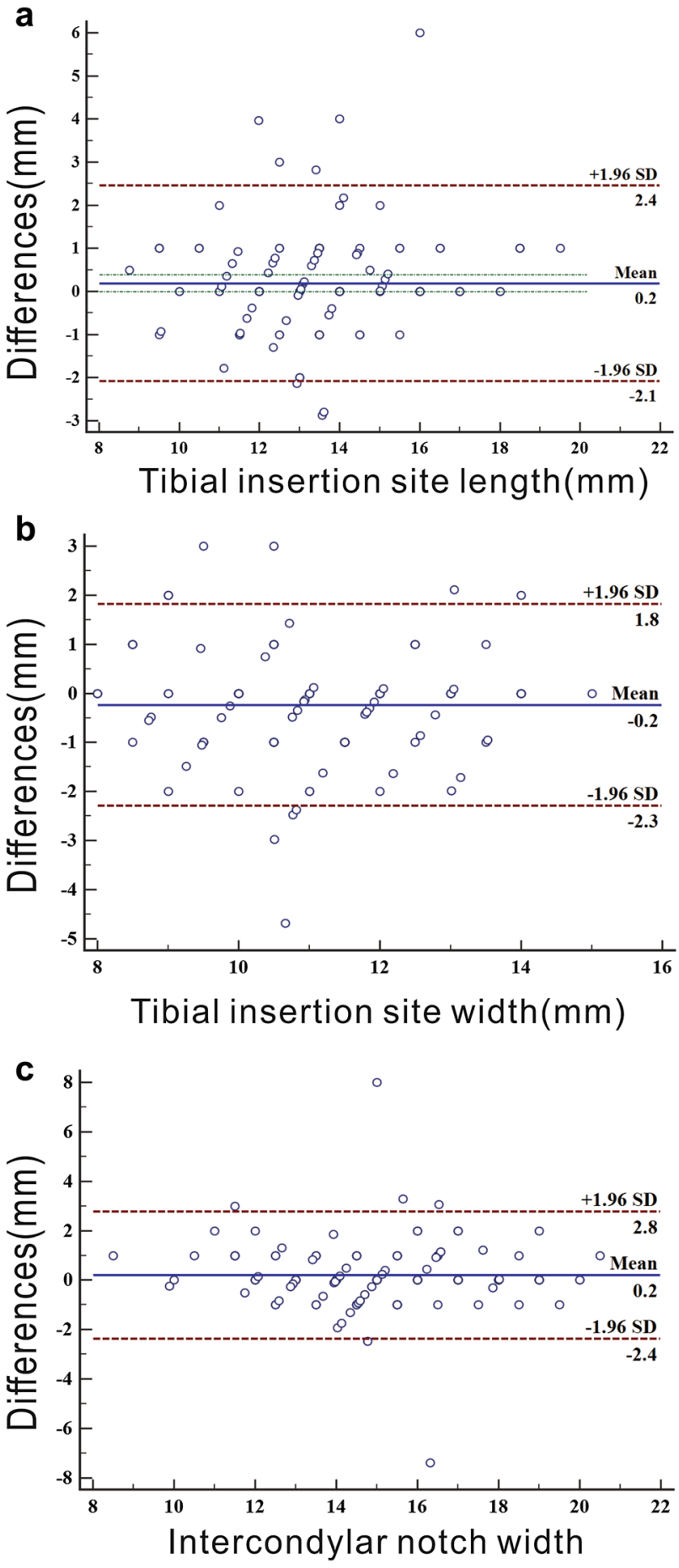

Fig. 2 Bland-Altman plot: agreement between the MRI and intraoperative measurements of tibial insertion site length, tibial insertion site width and intercondylar notch width. Values of 5.8\% (a, length of ACL insertion site), 6.5\% (b, width of ACL insertion site), and 4.3\% (c, width of intercondylar notch) are outside the limits of agreement
Table 3 Differences in the notch and insertion site characteristics between male and female subjects

\begin{tabular}{llll}
\hline & $\begin{array}{l}\text { Females }(n=35) \\
\text { Mean }\end{array}$ & $\begin{array}{l}\text { Males }(n=102) \\
\text { Mean }\end{array}$ & $p$ value \\
\hline Tibial insertion length & 12.9 & 13.8 & n.s \\
Tibial insertion width & 10.7 & 10.9 & n.s \\
Notch width & 14.5 & 15.5 & $<0.05$ \\
\hline
\end{tabular}
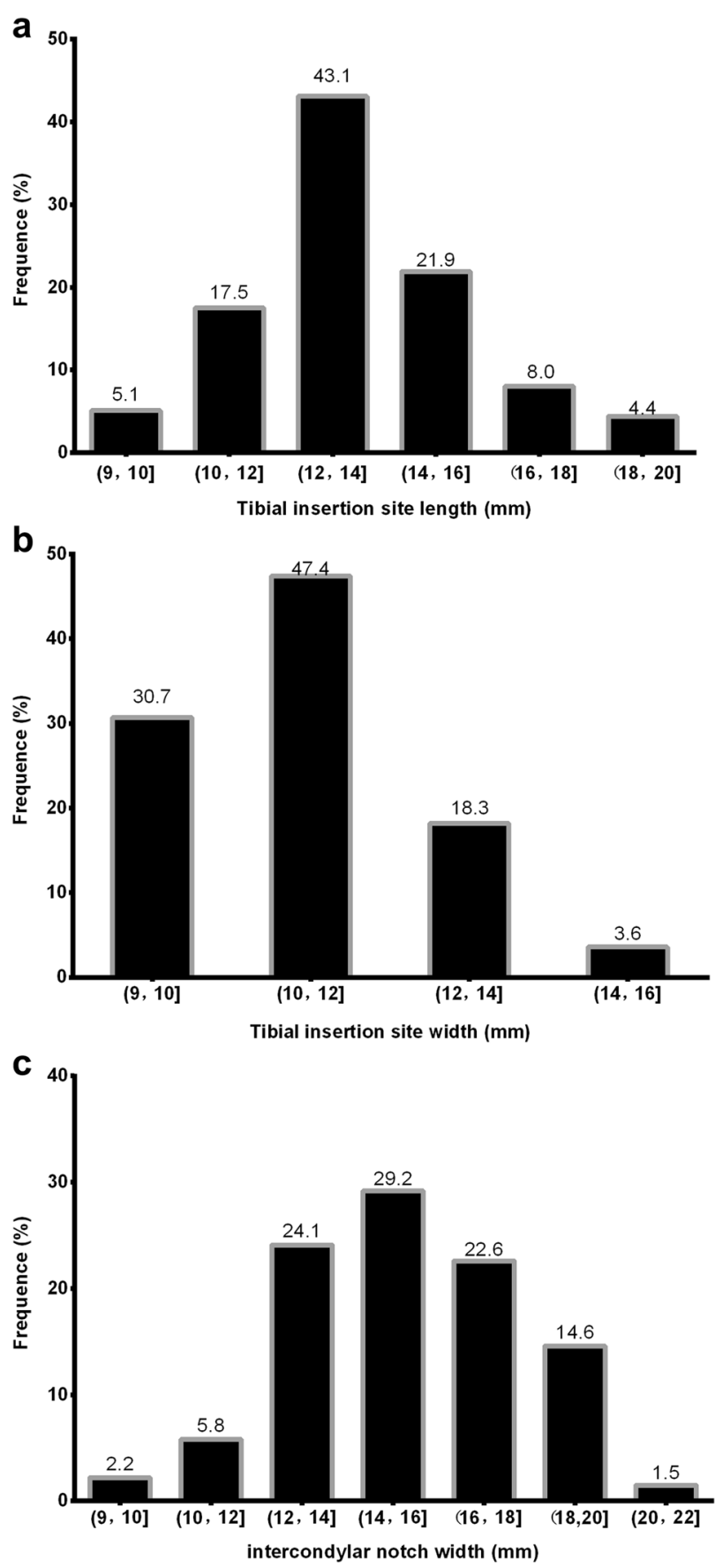

Fig. 3 The frequency direction of the tibial insertion site length and width and the intercondylar notch width 


\section{Discussion}

There are two important findings in the present study. First, the sizes of the tibial insertion of the ACL and the intercondylar notch width in Chinese patients with ACL injuries were significantly different compared to those in Western patients. Second, the number of individuals whose length of ACL tibial insertion was $<14 \mathrm{~mm}$ accounted for $65.7 \%$ of Chinese patients with ACL injuries.

It is imperative to perform preoperative anatomical studies because the results influence the optimal selection of grafts and reconstruction techniques [29, 43]. An extensive literature review has demonstrated great variability in the tibial insertion sizes of the ACL and the intercondylar notch $[8,11,13,15,16,20,22,24,36$, 42]. The length of the tibial ACL insertion sites reportedly varies from 9 to $38 \mathrm{~mm}$, while the insertion site width reportedly ranges from 4 to $10 \mathrm{~mm}[3,8,11,21$, 36]. Harald et al. [47] found a mean tibial insertion site length of $16.6 \pm 1.6 \mathrm{~mm}(11.9-21.0 \mathrm{~mm})$ as measured by MRI and a mean length of $16.4 \pm 1.6 \mathrm{~mm}(11.0-20.0 \mathrm{~mm})$ as assessed by intra-operative measurements using rulers in 146 patients who underwent primary ACL-R. Kopf et al. [22] documented that the tibial ACL insertion site had a mean length of $17.0 \pm 2.0 \mathrm{~mm}$ in 137 patients who underwent ACL-R as measured with an arthroscopic ruler. Femke et al. [43] reported that the length of the tibial ACL insertion site was $17 \pm 2.3 \mathrm{~mm}(12.0-22.0 \mathrm{~mm})$ and the width was $10 \pm 1.2 \mathrm{~mm}(7.0-14.0 \mathrm{~mm})$ in patients who underwent arthroscopic ACL-R. However, some findings showed that the tibial footprint size in Asians is smaller than that in Western populations [17, 23, 27]. A previous study reported that the average ACL tibial attachment length was $15.36 \pm 2.33 \mathrm{~mm}$ and the average width was $11.03 \pm 1.77 \mathrm{~mm}$ in 77 cadaveric knees of the Thai population as assessed by direct measurement [23]. Meanwhile, another study of 100 Japanese patients with knee pain who underwent radiography and MRI showed that the average ACL tibial length was $15.2 \pm 1.9 \mathrm{~mm}(14.8-15.5 \mathrm{~mm})$ [17]. South Korean researchers performed ACL tibial length intra-operative measurements in 127 patients who underwent primary total knee arthroplasty, and the average tibial footprint was $13.8 \mathrm{~mm}(10.0-18.0 \mathrm{~mm})$ in length and $9.8 \mathrm{~mm}(6.3-13.5 \mathrm{~mm})$ in width [27].

Intercondylar notch width also has significant variability and is affected by gender. Harner et al. [14] showed that the mean notch width in patients with non-contact ACL tears was $18.4 \mathrm{~mm}$. Femke et al. [48] examined patients undergoing ACL-R, and the notch width base was $16 \pm 2.9 \mathrm{~mm}(10-21 \mathrm{~mm})$. Similarly, Yang et al. found that the intercondylar notch width was $17.3 \pm 2.1 \mathrm{~mm}$ in 40 Chinese patients with unilateral ACL rupture [26]. In the largest series reviewed by Shelbourne et al., the mean notch width in 714 consecutive patients who underwent ACL-R was $13.9 \pm 2.2 \mathrm{~mm}$ for women and $15.9 \pm 2.5 \mathrm{~mm}$ for men [33]. Thus, the conclusion was that women have smaller notches than men do $[5,9,10,25,38,40]$. In another series, Shelbourne et al. and Joshua et al. also found variability between different ethnic groups, such as that African-American subjects have a larger mean intercondylar notch width than Caucasian subjects do $[12,34]$.

In those studies, the variation in insertion site sizes and intercondylar notch sizes may be a result of the age, gender, physique, differences in tissue quality, measuring techniques, and the race of the individuals. Iriuchishima et al. suggested that the differences in ACL size were more likely attributable to the generational differences in body size [19]. However, previous studies have failed to report exact findings in the Asian population. The available study results were limited by small sample sizes, the use of cadaveric specimens with degenerative changes, the inclusion of patients who did not receive ACL-R and measurements performed using a single technique. Therefore, these results were fundamentally flawed [18, 31, 32, 47]. To fill the knowledge gap, in addition to improving the number of research subjects and inclusion criteria, the measurements should also be carried out by different methods to obtain accurate results. Our research provides the desired data and found that the sizes of the ACL tibial insertion and intercondylar notch are relatively smaller among Chinese ACL injuries.

In the studies performed by the University of Pittsburgh, most of the ACL tibial insertion lengths among the patients undergoing ACL-R were between 16 and $18 \mathrm{~mm}$ [17, 22, 39, 43, 47]. Harald et al. [47] and Femke et al. [48] found lower proportions of ACL tibial insertion site length $<14 \mathrm{~mm}$ were $10 \%$ and $20 \%$, respectively, as assessed by MRI and an intra-operative ruler. In a study by Kopf et al. [22] $3.6 \%$ of knees were found to have ACL tibial insertion site lengths $<14 \mathrm{~mm}$. A total of $80.3 \%$ of knees found an ACL tibial insertion site length between 16 and $18 \mathrm{~mm}$. Mohsen et al. [39] determined the distributions of the total ACL insertion site length and found that $19.1 \%$ of patients had lengths less than $14 \mathrm{~mm}$, and $72.3 \%$ of patients had lengths between 16 and $18 \mathrm{~mm}$. However, Park et al. thought that the distribution of the ACL tibial footprint size widely varied in the patient population, and they reported a higher proportion (53.5\%) of tibial footprint length $<14 \mathrm{~mm}$ in South Korea females who underwent primary total knee arthroplasty [27]. Ichiba et al. [17] studied 100 Japanese patients with knee joint pain, and their report showed that the proportion of patients with ACL tibial footprint length $<14 \mathrm{~mm}$ was $28 \%$ as assessed via sagittal view on MRI. These findings showed that the proportion of tibial footprint size in Asians seems to be different from that in Western populations. However, there was no relative or exact research about the 
characteristics of ACL injuries among Asian populations. The present study confirms a higher proportion $(65.7 \%)$ of subjects with a tibial footprint size $<14 \mathrm{~mm}$ in the Chinese population of patients with ACL injuries.

$\mathrm{Fu}$ et al. suggested that a tibial insertion site length $<14 \mathrm{~mm}$ and notch width $<12 \mathrm{~mm}$ might be better reconstructed with an SB ACL-R [22, 41]. Siebold et al. suggested an SB ACL-R may be recommended for wide insertion sites up to $16 \mathrm{~mm}$ in length for complete footprint restoration [35, 37]. However, a small ACL tibial footprint can also undergo DB ACL-R. Chae et al. [4] described a surgical technique of DB ACL-R with a single tibia tunnel among 22 Korean patients with small ACL tibial footprints. The research showed significantly improved results. The size of the tunnel aperture is determined by the drill-guide angle and drill bit diameter, and the DB technique also requires consideration of a single graft size [20]. For ideal, individualized, anatomical ACL-R of Chinese ACL injuries, concerns exist regarding tibial bone-tunnel orientation, drill bit diameter, and SB graft size. Much care should be taken in the Chinese population when treating patients with a DB technique or larger graft options. Based on our results, such information about reconstructed individualized ACL ruptures in Chinese patients and decisions about the choice of graft in Chinese patients undergoing ACL-R may provide usefulness to surgeons.

A limiting factor is related to the preoperative MRI in the study. The MR images were acquired after the injury. Postinjury-associated changes may obscure tibial insertion site measurements. Another limiting factor pertains to the intraoperative measurements. The length of the insertion site was measured by the midcentral portal, and the real insertion site length might not always be accurately assessed. The loss of fibres caused by the injury may result in an underestimation of the insertion site measurement. However, our senior surgeon has a great deal of experience in identifying the ACL insertion site. Finally, MRI and intra-operative measurements were obtained only by a single observer, and we were unable to evaluate the intra- and inter-observer variability. Even so, we performed the measurements both on MRI and intra-operatively and avoided measurement and distortion errors through a standardized protocol. In addition, the measurements also showed a high confidence and accuracy. Based on the above analyses, we believed the measurements were authentic.

\section{Conclusion}

The results of the study showed the sizes of the ACL tibial insertion and intercondylar notch width in Chinese patients with ACL injuries. The data suggest that the proportion of the Chinese population with a small tibial footprint size seems different from that of the Western population. There is a higher proportion of subjects with a tibial footprint size $<14 \mathrm{~mm}$ in the Chinese population of patients with ACL injuries. Based on these anatomical characteristics, great care should be taken in the Chinese population when treating ACL rupture with a DB technique or larger graft options.

Acknowledgements The authors wish to thank Dr. Xiaonan Liu of the Anaesthesia Department at The First Affiliated Hospital of Chongqing Medical University for his assistance.

Author contributions Study design: NH, WH; Study conduct: NH, FL; Manuscript preparation and revision: NH, FL, SS; Data collection: FL, XG, ZL, LQ, JY, TW, ZH; Data analysis: FL, SZ; Figure preparation: FL; Data interpretation: NH, XL; Approval of the final version of manuscript: $\mathrm{NH}, \mathrm{WH}$; Funding acquisition: $\mathrm{NH}$.

Funding This work was supported by research Grants from the National Natural Science Foundation of China (NSFC) (Grant number 81672167$)$.

\section{Compliance with ethical standards}

Conflict of interest The authors declare that they have no competing interests.

Ethical approval Institutional Review Board (IRB) approval was obtained from the IRB (ID 2017-191) prior to the initiation of this study.

Informed consent Informed written consent was obtained from all study participants and their parents.

Open Access This article is distributed under the terms of the Creative Commons Attribution 4.0 International License (http://creativeco mmons.org/licenses/by/4.0/), which permits unrestricted use, distribution, and reproduction in any medium, provided you give appropriate credit to the original author(s) and the source, provide a link to the Creative Commons license, and indicate if changes were made.

\section{References}

1. Araujo P, van Eck CF, Torabi M, Fu FH (2013) How to optimize the use of MRI in anatomic ACL reconstruction. Knee Surg Sports Traumatol Arthrosc 21:1495-1501

2. Araujo PH, van Eck CF, Macalena JA, Fu FH (2011) Advances in the three-portal technique for anatomical single- or doublebundle ACL reconstruction. Knee Surg Sports Traumatol Arthrosc 19:1239-1242

3. Bellier G, Christel P, Colombet P, Djian P, Franceschi JP, Sbihi A (2004) Double-stranded hamstring graft for anterior cruciate ligament reconstruction. Arthroscopy 20:890-894

4. Chae IJ, Bae JH, Wang JH, Jeon J, Park JH (2013) Double-bundle anterior cruciate ligament reconstruction with split Achilles allograft and single tibia tunnel for small ACL tibial footprint: technical note with clinical results. Arch Orthop Trauma Surg 133:819-825 
5. Charlton WP, John TA, Ciccotti MG, Harrison N, Schweitzer M (2002) Differences in femoral notch anatomy between men and women: a magnetic resonance imaging study. Am J Sports Med 30:329-333

6. Chaudhari AM, Zelman EA, Flanigan DC, Kaeding CC, Nagaraja HN (2009) Anterior cruciate ligament-injured subjects have smaller anterior cruciate ligaments than matched controls: a magnetic resonance imaging study. Am J Sports Med 37:1282-1287

7. Chhabra A, Starman JS, Ferretti M, Vidal AF, Zantop T, Fu FH (2006) Anatomic, radiographic, biomechanical, and kinematic evaluation of the anterior cruciate ligament and its two functional bundles. J Bone Joint Surg Am 88(4):2-10

8. Colombet P, Robinson J, Christel P, Franceschi JP, Djian P, Bellier $G$ et al (2006) Morphology of anterior cruciate ligament attachments for anatomic reconstruction: a cadaveric dissection and radiographic study. Arthroscopy 22:984-992

9. Davis TJ, Shelbourne KD, Klootwyk TE (1999) Correlation of the intercondylar notch width of the femur to the width of the anterior and posterior cruciate ligaments. Knee Surg Sports Traumatol Arthrosc 7:209-214

10. Dienst M, Schneider G, Altmeyer K, Voelkering K, Georg T, Kramann B et al (2007) Correlation of intercondylar notch cross sections to the ACL size: a high resolution MR tomographic in vivo analysis. Arch Orthop Trauma Surg 127:253-260

11. Edwards A, Bull AM, Amis AA (2007) The attachments of the anteromedial and posterolateral fibre bundles of the anterior cruciate ligament: Part 1: tibial attachment. Knee Surg Sports Traumatol Arthrosc 15:1414-1421

12. Everhart JS, Flanigan DC, Chaudhari AM (2014) Anteromedial ridging of the femoral intercondylar notch: an anatomic study of 170 archival skeletal specimens. Knee Surg Sports Traumatol Arthrosc 22:80-87

13. Ferretti M, Ekdahl M, Shen W, Fu FH (2007) Osseous landmarks of the femoral attachment of the anterior cruciate ligament: an anatomic study. Arthroscopy 23:1218-1225

14. Harner CD, Paulos LE, Greenwald AE, Rosenberg TD, Cooley VC (1994) Detailed analysis of patients with bilateral anterior cruciate ligament injuries. Am J Sports Med 22:37-43

15. Hofbauer M, Muller B, Murawski CD, van Eck CF, Fu FH (2014) The concept of individualized anatomic anterior cruciate ligament (ACL) reconstruction. Knee Surg Sports Traumatol Arthrosc 22:979-986

16. Hwang MD, Piefer JW, Lubowitz JH (2012) Anterior cruciate ligament tibial footprint anatomy: systematic review of the 21 st century literature. Arthroscopy 28:728-734

17. Ichiba A, Kido H, Tokuyama F, Makuya K, Oda K (2014) Sagittal view of the tibial attachment of the anterior cruciate ligament on magnetic resonance imaging and the relationship between anterior cruciate ligament size and the physical characteristics of patients. J Orthop Sci 19:97-103

18. Illingworth KD, Hensler D, Working ZM, Macalena JA, Tashman S, Fu FH (2011) A simple evaluation of anterior cruciate ligament femoral tunnel position: the inclination angle and femoral tunnel angle. Am J Sports Med 39:2611-2618

19. Iriuchishima T, Ryu K, Fu FH (2018) Evaluation of age-related differences in anterior cruciate ligament size. Knee Surg Sports Traumatol Arthrosc;10.1007/s00167-018-5336-1

20. Kopf S, Martin DE, Tashman S, Fu FH (2010) Effect of tibial drill angles on bone tunnel aperture during anterior cruciate ligament reconstruction. J Bone Joint Surg Am 92:871-881

21. Kopf S, Musahl V, Tashman S, Szczodry M, Shen W, Fu FH (2009) A systematic review of the femoral origin and tibial insertion morphology of the ACL. Knee Surg Sports Traumatol Arthrosc 17:213-219
22. Kopf S, Pombo MW, Szczodry M, Irrgang JJ, Fu FH (2011) Size variability of the human anterior cruciate ligament insertion sites. Am J Sports Med 39:108-113

23. Kulkamthom N, Arkasihayuth A, Charakorn K, Chaimut M, Reeboonlap N (2012) The study of anterior cruciate ligament footprint in Thai population: a human cadaveric study. J Med Assoc Thai 95(10):S167-S172

24. Mochizuki T, Muneta T, Nagase T, Shirasawa S, Akita KI, Sekiya I (2006) Cadaveric knee observation study for describing anatomic femoral tunnel placement for two-bundle anterior cruciate ligament reconstruction. Arthroscopy 22:356-361

25. Murshed KA, Cicekcibasi AE, Karabacakoglu A, Seker M, Ziylan T (2005) Distal femur morphometry: a gender and bilateral comparative study using magnetic resonance imaging. Surg Radiol Anat 27:108-112

26. Ouyang X, Wang YH, Wang J, Hong SD, Xin F, Wang L et al (2016) MRI measurement on intercondylar notch after anterior cruciate ligament rupture and its correlation. Exp Ther Med 11:1275-1278

27. Park YB, Song YS, Kim SC, Park YG, Ha CW (2015) The size of tibial footprint of anterior cruciate ligament and association with physical characteristics in Asian females. Arch Orthop Trauma Surg 135:985-992

28. Pombo MW, Shen W, Fu FH (2008) Anatomic double-bundle anterior cruciate ligament reconstruction: where are we today? Arthroscopy 24:1168-1177

29. Rabuck SJ, Middleton KK, Maeda S, Fujimaki Y, Muller B, Araujo PH et al (2012) Individualized anatomic anterior cruciate ligament reconstruction. Arthrosc Tech 1:e23-e29

30. Rahnemai-Azar AA, Sabzevari S, Irarrazaval S, Chao T, Fu FH (2016) Anatomical individualized ACL reconstruction. Arch Bone Jt Surg 4:291-297

31. Schwartzberg R, Snyder K, Reuss B (2015) Preoperative measurement of ACL insertion sites. J Knee Surg 28:89-94

32. Shea KG, Apel PJ, Pfeiffer RP, Showalter LD, Traughber PD (2002) The tibial attachment of the anterior cruciate ligament in children and adolescents: analysis of magnetic resonance imaging. Knee Surg Sports Traumatol Arthrosc 10:102-108

33. Shelbourne KD, Davis TJ, Klootwyk TE (1998) The relationship between intercondylar notch width of the femur and the incidence of anterior cruciate ligament tears. A prospective study. Am J Sports Med 26:402-408

34. Shelbourne KD, Gray T, Benner RW (2007) Intercondylar notch width measurement differences between African American and white men and women with intact anterior cruciate ligament knees. Am J Sports Med 35:1304-1307

35. Siebold R (2011) The concept of complete footprint restoration with guidelines for single- and double-bundle ACL reconstruction. Knee Surg Sports Traumatol Arthrosc 19:699-706

36. Siebold R, Ellert T, Metz S, Metz J (2008) Tibial insertions of the anteromedial and posterolateral bundles of the anterior cruciate ligament: morphometry, arthroscopic landmarks, and orientation model for bone tunnel placement. Arthroscopy 24:154-161

37. Siebold R, Schuhmacher P (2012) Restoration of the tibial ACL footprint area and geometry using the modified insertion site table. Knee Surg Sports Traumatol Arthrosc 20:1845-1849

38. Stijak L, Radonjic V, Nikolic V, Blagojevic Z, Aksic M, Filipovic B (2009) Correlation between the morphometric parameters of the anterior cruciate ligament and the intercondylar width: gender and age differences. Knee Surg Sports Traumatol Arthrosc 17:812-817

39. Takahashi M, Doi M, Abe M, Suzuki D, Nagano A (2006) Anatomical study of the femoral and tibial insertions of the anteromedial and posterolateral bundles of human anterior cruciate ligament. Am J Sports Med 34:787-792 
40. Tillman MD, Smith KR, Bauer JA, Cauraugh JH, Falsetti AB, Pattishall JL (2002) Differences in three intercondylar notch geometry indices between males and females: a cadaver study. Knee 9:41-46

41. van Eck CF, Lesniak BP, Schreiber VM, Fu FH (2010) Anatomic single- and double-bundle anterior cruciate ligament reconstruction flowchart. Arthroscopy 26:258-268

42. van Eck CF, Martins CA, Vyas SM, Celentano U, van Dijk CN, Fu FH (2010) Femoral intercondylar notch shape and dimensions in ACL-injured patients. Knee Surg Sports Traumatol Arthrosc $18: 1257-1262$

43. van Eck CF, Schreiber VM, Liu TT, Fu FH (2010) The anatomic approach to primary, revision and augmentation anterior cruciate ligament reconstruction. Knee Surg Sports Traumatol Arthrosc 18:1154-1163

44. van Eck CF, Widhalm H, Murawski C, Fu FH (2015) Individualized anatomic anterior cruciate ligament reconstruction. Phys Sportsmed 43:87-92

45. Whitney DC, Sturnick DR, Vacek PM, DeSarno MJ, GardnerMorse M, Tourville TW et al (2014) Relationship between the risk of suffering a first-time noncontact ACL injury and geometry of the femoral notch and ACL: a prospective cohort study with a nested case-control analysis. Am J Sports Med 42:1796-1805

46. Widhalm HK, Surer L, Kurapati N, Guglielmino C, Irrgang JJ, Fu FH (2014) Tibial ACL insertion site length: correlation between preoperative MRI and intra-operative measurements. Knee Surg Sports Traumatol Arthrosc. https://doi.org/10.1007/s0016 7-014-3473-8

47. Widhalm HK, Surer L, Kurapati N, Guglielmino C, Irrgang JJ, Fu FH (2016) Tibial ACL insertion site length: correlation between preoperative MRI and intra-operative measurements. Knee Surg Sports Traumatol Arthrosc 24:2787-2793

48. Wolters F, Vrooijink SH, Van Eck CF, Fu FH (2011) Does notch size predict ACL insertion site size? Knee Surg Sports Traumatol Arthrosc 19(1):S17-S21

49. Zantop T, Petersen W, Sekiya JK, Musahl V, Fu FH (2006) Anterior cruciate ligament anatomy and function relating to anatomical reconstruction. Knee Surg Sports Traumatol Arthrosc 14:982-992 